\title{
Diffusion Model Based on Cellular Automata and Its Application
}

\author{
Jiaoyan $\mathrm{Ai}^{1, \mathrm{a}}$, Sizhi Wu${ }^{1, \mathrm{~b}}$, Zongming Wei ${ }^{1, \mathrm{c}}$, Haiyang $\mathrm{Xu}^{1, \mathrm{~d}}$ and Gang Liu ${ }^{1, \mathrm{e}}$ \\ ${ }^{1}$ Electrical Engineering College, Guangxi University, Nanning 530004, China. \\ aaijy@gxu.edu.cn , 'busizhi1989@163.com, 'wei943718889ted@hotmail.com, \\ d554001527@qq.com, e672436229@qq.com
}

Keywords: Cellular Automata, Diffusion Model, Dynamic Spatial-temporal Simulation.

\begin{abstract}
We adopt cellular automata, a kind of complex system modeling method which achieves macro-emergence through local transition rules, to build a diffusion model. We realize parallel computation of the model with MATLAB's matrix processing and develop a GUI for model running. Through adjusting the diffusion parameters, diffusion pattern parameters, diffusion control parameters with the GUI, we achieve dynamic simulation of various diffusions' spatial-temporal changing. Apply this model to simulate air pollution diffusing and visualize simulation results in GIS. The results show that the model can not only dynamically simulate diffusion models, but also simulate diffusion under unsteady atmosphere (such as changed wind direction and speed or turbulent flow) by reasonable parameter settings. With accurate dynamic simulation of spatial-temporal distribution of pollution the model is supposed to be feasible and reasonable, and is expected to be used to simulate and predict the various diffusion patterns.
\end{abstract}

\section{Introduction}

In nature, diffusion refers to the phenomenon that molecules transfer from regions of higher concentration to lower concentration, such as pollutants diffuse in the air, water, soil, etc. Diffusion phenomenon is a complex process with dynamic changes of spatial-temporal. Currently, we lack a practical model to reflect the dynamic mode accurately. Cellular automaton is an achievement with the development of computer science and life science. Cellular automata is capable of simulating objects' complex motion. Based on the local transformation rules between cellular and its neighbors, it could simulate the macroscopic phenomena of entire space. In natural environment, the local transfer rules of diffusion phenomenon can be described with diffusion coefficients in different directions. Cellular Automata is often selected by researchers as a way of modeling to analyze other complex systems, such as the formation of snowflakes, fluid, turbulent motion [1-5], etc. Therefore, we use cellular automata to build a diffusion model and apply it to simulate the diffusion of air pollution.

\section{Cellular Automata Model of Diffusion}
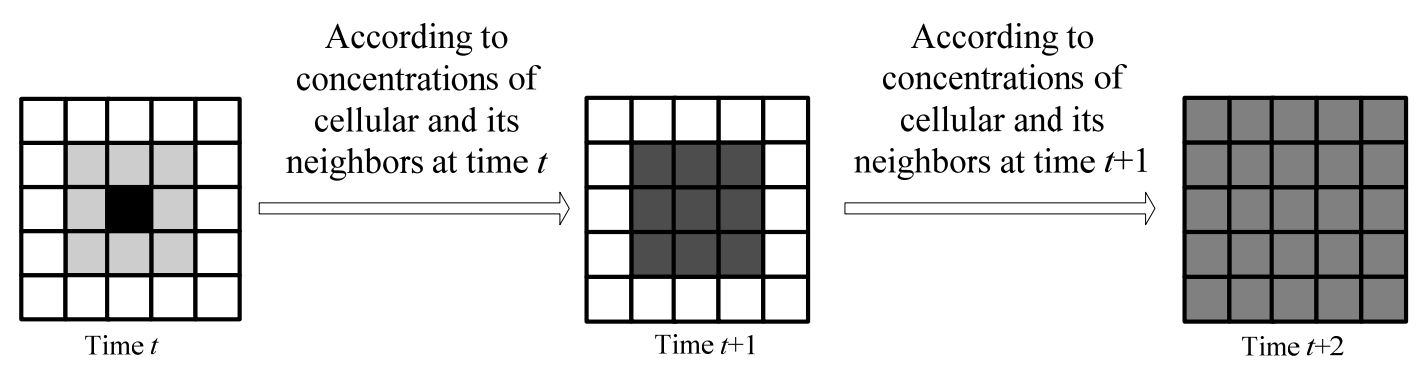

Fig.1 The structure of the cellular automaton

Based on the theory of Cellular Automata, we build cellular automata model of the diffusion (see Fig.1), the model includes cellular, cellular space, cellular state and state transition rule. We use the two-dimensional matrix as cellular space and two-dimensional matrix elements as cellular automata. 
The currently concentration is completely determined by the previous concentration of the cell and its neighbors with state transition rule [6,7]. Dispersion modeling of two-dimensional cellular automata diffusion model established in this paper is shown in Fig.1.

The neighbors of the cellular in eight directions are up-cellular, down-cellular, left-cellular, right-cellular, left-up-cellular, left-down-cellular, right-up-cellular and right-down-cellular. According to the concentration of pollutants diffusion rule, cellular state transition rule is defined as Eq.1 [8].First, we determine the cellular concentration of pollutants, and divide them into several levels by the range of the concentration.

$$
\left\{\begin{aligned}
E_{i, j}^{t+1}= & E_{i, j}^{t}+P_{u p}^{t}\left(E_{i-1, j}^{t}-E_{i, j}^{t}\right)+P_{\text {down }}^{t}\left(E_{i, j-1}^{t}-E_{i, j}^{t}\right)+P_{\text {left }}^{t}\left(E_{i, j-1}^{t}-E_{i, j}^{t}\right) \\
& +P_{\text {right }}^{t}\left(E_{i, j+1}^{t}-E_{i, j}^{t}\right)+P_{\text {left-up }}^{t}\left(E_{i-1, j-1}^{t}-E_{i, j}^{t}\right)+P_{\text {left-down }}^{t}\left(E_{i+1, j-1}^{t}-E_{i, j}^{t}\right) \\
& +P_{\text {right-up }}^{t}\left(E_{i+1, j-1}^{t}-E_{i, j}^{t}\right)+P_{\text {right-down }}^{t}\left(E_{i+1, j+1}^{t}-E_{i, j}^{t}\right) \\
S_{i, j}^{t+1}= & s_{1}, E_{i, j}^{t+1} \leq \text { threshold } 1 \\
S_{i, j}^{t+1}= & s_{2}, \text { threshold } 1 \leq E_{i, j}^{t+1} \leq \text { threshold } 2 \\
S_{i, j}^{t+1}= & s_{3}, \text { threshold } 2 \leq E_{i, j}^{t+1} \leq \text { threshold } 3 \\
S_{i, j}^{t+1}= & s_{4}, \text { threshold } 3 \leq E_{i, j}^{t+1} \leq \text { threshold } 4 \\
S_{i, j}^{t+1}= & s_{5}, \text { threshold } 4 \leq E_{i, j}^{t+1}
\end{aligned}\right.
$$

In the expression, $E_{i, j}^{t}$ is the pollutant concentration of cellular $(i, j)$ at time t. $E_{i, j-1}^{t} 、 E_{i, j+1}^{t} 、 E_{i-1, j}^{t}$ and $E_{i+1, j}^{t}$ are the concentration of left-cellular, right-cellular, up-cellular and down-cellular, respectively. $E_{i-1, j-1}^{t} 、 E_{i+1, j-1}^{t} 、 E_{i-1, j+1}^{t}$ and $E_{i+1, j+1}^{t}$ are the concentration of left-up-cellular, left-down-cellular, right-up-cellular, and right-down-cellular. $E_{i, j}^{t+1}$ is the concentration of cellular ( $i$, $j$ ) at time $t+1$, which equals to the sum of each difference between $E_{i, j}^{t}$ and concentration of its neighbors multiply by corresponding diffusion coefficient. $P_{m}^{t}$ is the transfer coefficient of pollutant concentration between the cellular and its neighbors, which depends on pollutant diffusion coefficient, wind speed and direction, etc. Cellular concentrations are divided into five levels $\left\{s_{1}, s_{2}, s_{3}, s_{4}, s_{5}\right\}$, which are represented by the set of colors as $\{$ white, gray-1, gray-2, gray-3, black $\}$. The $\{$ threshold1, threshold2, threshold3, threshold4 $\}$ is $\{0.1,0.3,0.6,0.9\}$. The study showed that the trend of air pollution diffusion is mainly affected by wind speed and wind direction [9].

For up-cellular, down-cellular, left-cellular and right-cellular, $P_{m}^{t}$ refer with Eq.2.

$P_{m}^{t}=w * v_{t}+d$.

For left-up-cellular, left-down-cellular, right-up-cellular and right-down-cellular, $P_{m}^{t}$ refer with Eq.3.

$P_{m}^{t}=0.1 * w * v_{i}+d$.

In Eq.2 and Eq.3, $w$ is factor of wind, $v_{i}$ is the wind speed at current time, $d$ is diffusion coefficient of itself.

\section{Model's Realization}

We realize parallel computation of the model with MATLAB's matrix processing and develop a GUI for model running.

Build Cellular Automata Model. MATLAB has capabilities of fast matrix operations and powerful visualization. It is suitable for research and development and application of algorithms. We build cellular automata model of diffusion in Matlab-R2012b. The steps are as follows:

At first, establish a two-dimensional matrix to represent the two-dimensional cellular space, matrix element represents cellular, the values of elements indicate cellular concentration, and the 
index number of matrix element corresponds to cellular position, the location of sources and pollutant concentrations are initialized. The second step is to calculate the concentration of cellular pollutant $E_{i, j}^{t+1}$ and confirm its status by Eq.1. Then, according to the threshold range where the states values of each cellular lies, we can determine its cellular states levels. The third step, make use of MATLAB's graphics display to visualize the cellular space by its state level. Finally, comparing the times $t$ of diffusion and the set point $s$, if $\mathrm{t}<\mathrm{s}$, the model continue to run from the second step, otherwise, stop it. As shown in Fig.2.

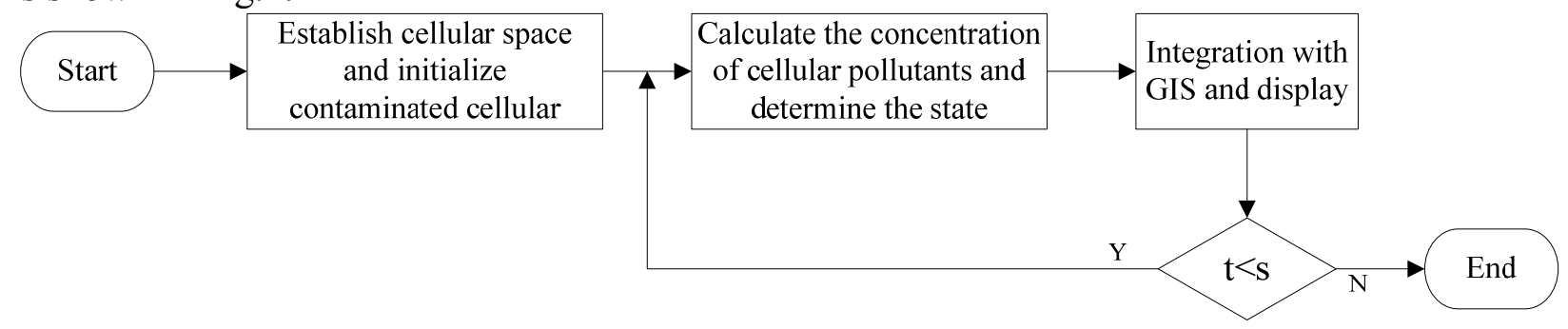

Fig.2 Simulation process

GUI of Diffusion Model. In order to facilitate the research and application, we developed a GUI for cellular automata diffusion model by MATLAB GUIDE. By setting the parameters and selecting diffusion mode, the mode can realize the simulation of multi-pollutant sources instantaneous diffusion, continuous diffusion, turbulence diffusion and dynamic wind direction of diffusion. The GUI uses a timer to control the diffusion process to reflect its dynamic characteristics. The development of GUI consists of three parts, which can be listed as follows, control and operation of the timer, read-and-call of parameters and display of diffusion. Operational procedure of the GUI is as follows: (1) Check the parameters are reasonable or not, then run the timer by clicking the start button, (2) Execute the callback function of the timer, confirm cellular status and visualize it, (3) Compare the run times and the set point, if they are equal, the program will terminate. As shown in Fig.3.

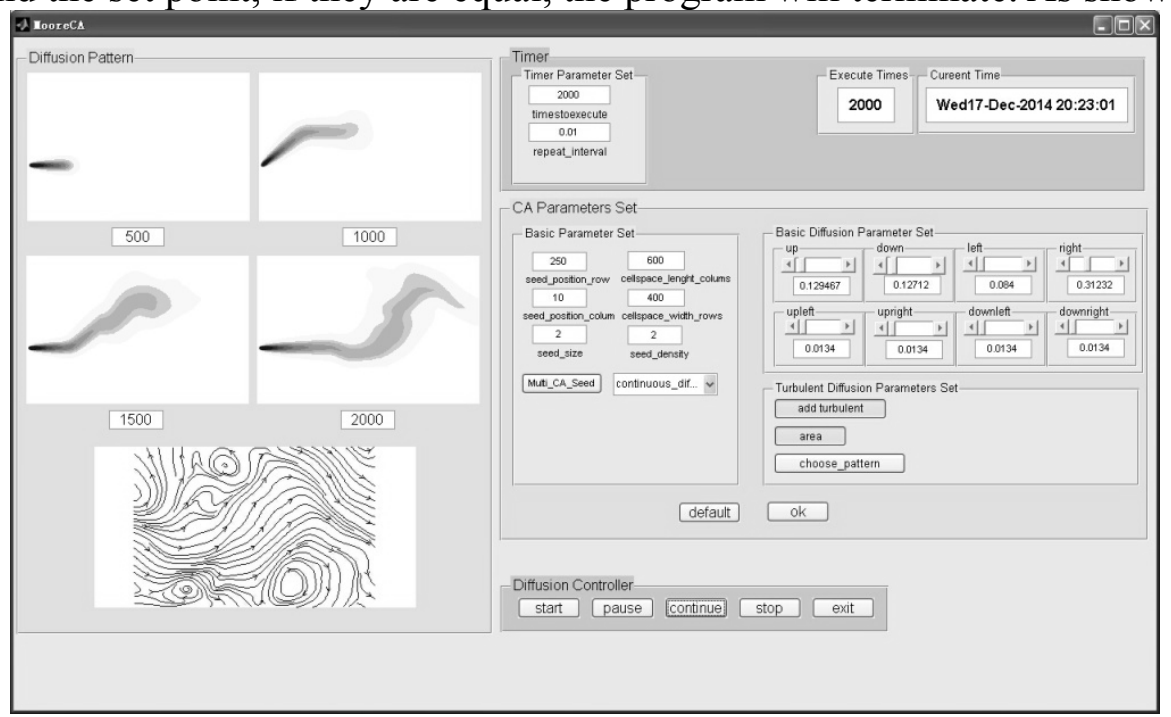

Fig.3 GUI and simulation result

According to the above, we developed the GUI by MATLAB GUIDE. As shown in Fig.3. The GUI is divided into 3 modules: parameters setting module, timer control module and diffusion visualization module. The parameters setting module includes cellular space size, position and concentration of pollution sources. The timer control module includes running times of program, the operation cycle of the program and interrupt and restart of program. The diffusion visualization module includes display of mimic diagram, display of running times and display of current time.

\section{Simulation and Results Analysis}

Air pollutant diffusion is sustainable and can be easily influenced by the weather. Its diffusion process is complex, so we take it as a simulation object. Suppose that it's a continuous diffusion, and 
the cellular space is $400 * 600$, intensity of pollution sources is 2 , and position of the diffusion source at the coordinates $(250,10)$ with wind speed of $15 \mathrm{~m} / \mathrm{s}$. It's a easterly wind at time $t_{1}$, then it changes to northeaster at time $t_{2}$ and changes to northerly at time $t_{3}$. After that, there is a turbulence at time $t_{4}$.

At time $t_{1}$, based on Eq.1, Eq.2 and Eq.3, we can get the diffusion coefficient of \{ up-cellular, down-cellular, left-cellular, right-cellular $\}$ and \{up-left-cellular, up-right-cellular, down-left-cellular, down-right-cellular $\}$ are $\{0.096,0.096,0.096,0.37\}$ and $\{0.014,0.014$, $0.014,0.026\}$, respectively. At time $t_{2}$, the \{ up-cellular, down-cellular, left-cellular, right-cellular $\}$ and $\{$ up-left-cellular, up-right-cellular, down-left-cellular, down-right-cellular $\}$ are $\{0.37,0.096$, $0.096,0.37\}$ and $\{0.014,0.014,0.014,0.026\}$, respectively. At time $t_{3}$, the $\{$ up-cellular, down-cellular, left-cellular, right-cellular $\}$ and \{up-left-cellular, up-right-cellular, down-left-cellular, down-right-cellular $\}$ are $\{0.37,0.096,0.096,0.097\}$ and $\{0.026,0.014,0.014$, $0.014\}$, respectively. At time $t_{4}$, there is a turbulence at right of the cellular space. The diffusion parameters will be changed with the trend of turbulence, when the pollutant enters the turbulence area.

According to the above, we change the parameters of cellular space, cellular diffusion and the timer at different times. At time $t_{1}$, the procedure starts to run, and the wind direction of pollution source is east. Then change the wind direction to north-east at time $t_{2}$ (run the procedure 500 times). At time $t_{3}$ (run the procedure 1000 times), change the wind direction to east and in the right side of the cellular space a turbulence occurs. The result of diffusion simulation is shown in Fig.3.

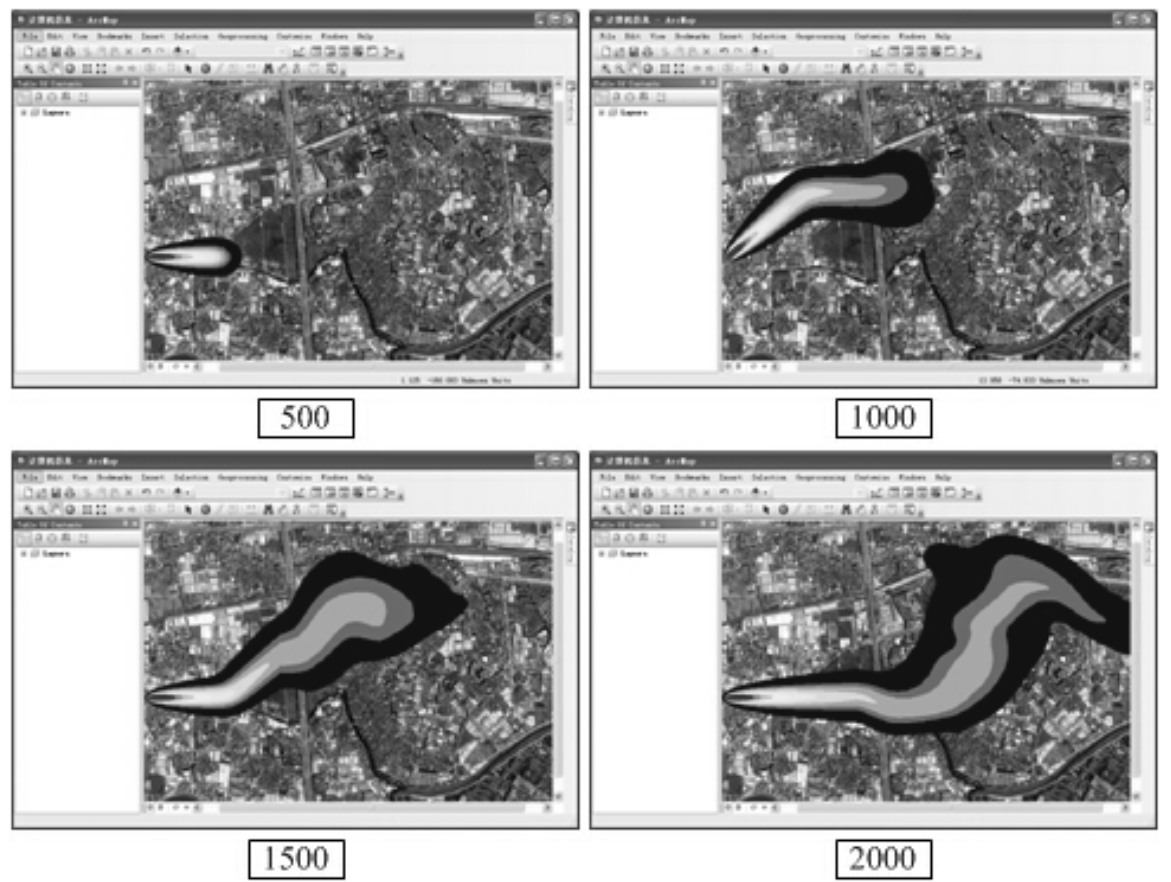

Fig.4 Visualization of simulation results in GIS

According to the Fig.3, the direction of pollutant diffusion changes accordingly as the wind direction changes. Between the time $t_{1}$ and $t_{2}$ (run the procedure from 0 to 500 times), the pollutants move to east with the wind, between time $t_{2}$ and $t_{3}$ (run the procedure from 500 times to 1000 times), the pollutants move to northeast with the wind. After time $t_{3}$, the pollutants move to east, but when these pollutants enter the turbulence area in the right of space, the diffusion direction changes with the trend of turbulence. In order to confirm the location of pollutants on an actual map, we visualize the results of simulation by GIS software, as shown in Fig.4. 


\section{Summary}

We build diffusion mode based on cellular automata and develop a GUI for it by MATLAB, what's more, we apply the model to simulate air pollutant diffusion. It proves that the model can simulate irregular diffusion under conditions of dynamic speeds and directions of wind and the turbulence, etc. By combing with GIS and other monitoring systems, we can find the factors which influence the process of diffusion from geographic database and real-time monitoring data, as well as the relationship between those factors. As a result, we can get accurate rules of dynamic local diffusion. We expect to use the model to simulate a variety of spatial-temporal dynamic diffusion realistically, and to provide a reasonable and credible basis for analysis and decision.

\section{Acknowledgement}

This research was financially supported by Science and Technology Plan Chairman Foundation of Guangxi (1517-08). The corresponding author of this paper is Ai Jiaoyan, a professor at Guangxi University.

\section{References}

[1] Duan Xiaodong, Wang Cunrui, Liu Xiangdong. Research and Simulation of the cellular automaton, Beujing: Science Publishing Company, 2012.

[2] Zhou Chenghu, Sun Zhanli, Xie Yichun. Study of geographical cellular automaton, Beujing: Science Publishing Company, 1999.

[3] John E, Hopcroft Rajeev Motwani Jeffrey D.Ullman. Introduction to automaton theory, language and calculation. Liu Tian, Jiang Hui, Wang Hanpin, translate, 2004.

[4] Wolfram S. A New Kind of Science. Michigan: Wolfram Media, 2002.

[5] Li Caiwei. Temporal-spatial simulation of the cellular automaton and complex system. Wuhan: Huazhong University of Science and Technology, 1997.

[6] Niloy Ganguly. Cellular Automata Evolution: Theory and Applications in Pattern Recognition and Classification. India: A Deemed University, 2003.

[7] Sarkar P. A Brief History of Cellular Automata. ACM Computing Systems, 2000, 32 (1): 80 - 107.

[8] Loannis Karafyllidis. A model for the prediction of oil slick movement and spreading using cellular automata. Environment International, 1997, 23(6): 839 - 850.

[9] Ping Cuo. Study on the application of air pollution diffusion model in long term. Tianjin University, 2006. 